\title{
Anti-NY-ESO1/LAGE-1A TCR/scFv Anti-CD3 IMCnyeso
}

National Cancer Institute

\section{Source}

National Cancer Institute. Anti-NY-ESO1/LAGE-1A TCR/SCFV Anti-CD3 IMCnyeso. NCI

Thesaurus. Code C153130.

A bispecific molecule composed of a soluble, affinity-enhanced T-cell receptor (TCR) specific for human leukocyte antigen A2 (HLA-A2)-restricted cancer-testis antigens (CTAs) NY-ESO-1 and L antigen family member 1 isoform A (LAGE-1A; LAGE-A1; CT6.2a), fused to a single-chain variable fragment (scFv) specific for the T-cell surface antigen CD3, with potential immunomodulating and antineoplastic activities. Upon infusion, antiNY-ESO1/LAGE-1A TCR/scFv anti-CD3 IMCnyeso specifically targets and binds with its TCR moiety to NY-ESO-1 and/or LAGE-1A expressed on tumor cells and with its scFv moiety to CD3 expressed on T-cells. This crosslinks tumor cells and T-cells, re-directs and activates T-cells, and results in a cytotoxic T-lymphocyte (CT L)-mediated destruction of NY-ESO-1 and/or LAGE-1A-positive tumor cells. NY-ESO-1 and LAGE-1A, members of the cancer-testis antigen (CTA) family, are overexpressed on the surface of various tumor cell types; they share a specific HLA-A*0201 epitope, 157-165, which is expressed on certain tumor cell types while its expression is not found on normal, healthy cells. 